\title{
Desk of the Editor Vol.8 Issue 1
}

\author{
K. S. Gopinath ${ }^{1}$
}

Published online: 24 December 2016

(C) Indian Association of Surgical Oncology 2016

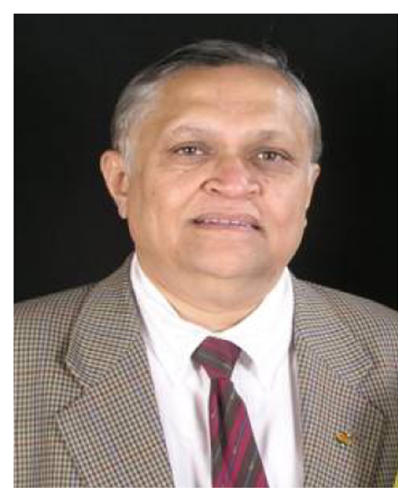

Seasons greetings and Happy New Year 2017.

As I write this editorial, the year 2016 is coming to an end and it is the dawn of another exciting year. There is a big challenge in 2017 for IJSO and IASO. IASO being partner of the mega scientific event-The 2nd Indian Cancer Congress 2017 to be held in Bengaluru.

Being the partner association, it is my duty to spread the message to its' members to participate in the scientific programme by submitting their work. Which is the need of the hour for members, to the association and the Journal.

The theme of the conference is "Insight, Innovation and Integration". I appeal to all the contributing authors,

K. S. Gopinath

gopiijso@gmail.com

1 HCG - Bangalore Institute of Oncology, 44/45 Raja Ram Mohan Roy Extension, Off K H Double Road, Bangalore 560027, India

reviewers, editorial board members and the committee of the IASO to take note of the theme and plan the scientific programme for IASO.

I thank the committee of IASO and the members who reposed faith in me and honored me by electing me for a another term as chairman/Editor of this unique journal. There is more responsibility given to me to take it forward and improve the impact factor of the journal. This is only possible if all members actively contribute their work.

In spite of mushrooming of many open access journal, our journal has progressed slowly, steadily, and ethically. The editorial board has put all efforts to take it forward and make it a journal with a difference. Recently ASCO post has reviewed our journal and written an article appreciating the publication of the journal in spite of hardships in this part of the world.

This issue of the journal highlights the changing trend in management of Urological Malignancies keeping in mind advances made in understanding Biology, Genomics, Target therapies and Precision tools like Minimal Invasive Surgery, Radio Genomics and Radiation treatment Protocols. You are all aware there is a changing trend in Epidemiological data which shows Prostate is taking over other malignances like Lung and Head and Neck Malignances in Men in the Indian sub continent. The issue highlights changing trend from Nephrectomy to Nephron sparing procedure by Shree Prasad et al... \& Venkat Ramani et al... advances in metastatic renal disease with usefulness of target therapy has been brought out well by review article by Amit Joshi et al...., Konnety et al... highlighted the management of Muscle invasive bladder cancer. 
The preface note by Prof. Kulkarni has shown how Uro oncology as a specialty has developed in the Indian sub continent which has resulted in high quality care and good out comes for urological malignancies.

The Ronald Piana of ASCO post reviewed the performance of the journal and published about IJSO in the Global Oncology section.
Finally I thank the Editorial board, Executive committee members of IASO and Dr. K.Harish, Dr. Chintamani, Dr. Sanjeev Misra and Mr. Suresha J (Dayananda Sagar Institution), for their continuous support for bringing out the journal in time. Special thanks to our publishers Team Springer. 